\title{
Carnets
}

Revue électronique d'études françaises de l'APEF

Deuxième série - 1 | 2014

L'étranger

\section{Étrangeté et étranger dans l'univers de Philippe Claudel}

\section{Dominique Bonnet}

\section{(2) OpenEdition}

Journals

\section{Édition électronique}

URL : http://journals.openedition.org/carnets/1145

DOI : 10.4000/carnets. 1145

ISSN : 1646-7698

Éditeur

APEF

\section{Référence électronique}

Dominique Bonnet, «Étrangeté et étranger dans l'univers de Philippe Claudel », Carnets [En ligne], Deuxième série - 1 | 2014, mis en ligne le 30 mai 2014, consulté le 19 avril 2019. URL : http:// journals.openedition.org/carnets/1145; DOI : 10.4000/carnets.1145

Ce document a été généré automatiquement le 19 avril 2019.

\section{(c) (i) (8)}

Carnets est mis à disposition selon les termes de la licence Creative Commons - Atribution - Pas d'utilisation commerciale 4.0 International. 


\title{
Étrangeté et étranger dans l'univers de Philippe Claudel
}

\section{Dominique Bonnet}

\author{
« On définit souvent l'individualité comme ce qui \\ constitue l'originalité d'un individu, par définition \\ "indivisible", et ce qui lui appartient en propre, en \\ l'opposant pour cela à des formes sociales \\ collectives comme la société ou les classes \\ sociales » (Fugier, 2006 :2)
}

\section{Introduction}

1 Le rapport de Brodeck de Philippe Claudel, dont nous allons parler dans cet article, nous semble constituer, de par sa construction, une véritable mise en abîme de l'étranger et de l'étrange face à la communauté. L'originalité de l'individu pour reprendre le terme de Pascal Fugier est à plusieurs reprises mise en avant par Philippe Claudel comme une des causes de son rejet par la communauté.

2 Brodeck a passé une grande partie de sa vie dans un village frontalier. Contre son gré il sera choisi par le reste de la communauté villageoise pour rédiger un rapport sur un évènement dramatique. À travers l'écriture forcée de ce rapport sur le lynchage d'un étranger, l'Anderer (l'autre en allemand), par le reste des villageois, Brodeck se replonge dans son passé proche, dans sa souffrance dans l'univers des camps, cet univers dont on ne revient pas mais d'où lui, Brodeck, est revenu, retour de sa propre expérience de la xénophobie. De la sorte, dans l'écriture de Brodeck, le texte anonyme rejoint la confession intime et nous guide vers la douleur de l'indicible puis vers son apaisement par l'écriture de la survie, de la parabole, du conte dans lequel la loi naturelle élimine l'autre. Dans cet exercice de narration nous contemplons avec horreur jusqu'où peut mener la peur de la différence, la peur de l'Anderer c'est-à-dire la peur de l'autre, celui qui n'a plus de patrie, celui qui s'est perdu dans l'Histoire, qui ne retrouve plus son chemin et qui finit par être rejeté par ses semblables, terrible vécu de Brodeck. 
3 Afin d'étudier cette exclusion cyclique à laquelle nous assistons dans l'histoire créée par Claudel dans son rapport, nous reviendrons sur l'existence et le parcours de trois de ces personnages : Fédorine, Brodeck et L’Anderer.

\section{Fédorine ou l'étrangeté comme salvation}

4 Fédorine, mère adoptive de Brodeck, reste dans l'ombre tout au long du roman. C'est pourtant grâce à elle que Brodeck est vivant et qu'Émelia la femme de Brodeck a surmonté son douloureux passé.

5 Sur Fédorine rien ne nous est dit, ou presque. Lorsque Brodeck tente de parler d'elle, il est lui aussi indécis quant à son âge, son passé, son existence avant qu'il ne la connaisse : « Je ne sais pas si Fédorine a connu la jeunesse. Je l'ai toujours vue tordue et courbée, tavelée comme une nèfle oubliée trois saisons dans le cellier. Même lorsque j'étais un enfant et qu'elle m'a recueilli, elle ressemblait déjà à une sorcière cabossée » (Claudel, $2010: 28$ ). Brodeck semble connaître Fédorine depuis toujours, et pourtant il ne sait pratiquement rien d'elle. Dès sa plus jeune enfance Brodeck entre en contact avec l'étranger mais aussi avec l'étrangeté par Fédorine qui parle une langue qu'il ne comprend pas. Elle le recueille dans un village en ruine et l'emmène pour le sauver, pour lui donner une chance, qu'ellemême peut-être recherche dans cette fuite en charrette :

Elle venait de très loin, de très loin dans le temps, et de très loin dans la géographie des mondes. Elle s'était échappée du ventre pourri de l'Europe... C'était il y a longtemps : j'étais devant une maison en ruine qui fumait un peu. Peut-être était-ce celle de mon père, celle de ma mère? Je devais bien moi aussi avoir une famille. J'étais seul du haut de mes quatre ans... Fédorine est passée en tirant sa charrette. Elle m'a vu. Elle s'est arrêtée. Elle a fouillé dans sa besace pour en sortir une pomme d'un beau rouge luisant. Elle me l'a tendue... Fédorine m'a parlé, m'a dit des mots que je ne comprenais pas, et posé des questions auxquelles je n'ai pas su répondre, elle m'a touché le front et les cheveux (Ibid.)

6 Fédorine est déjà elle-même une étrangère dans cette société qu'elle traverse, dans ce monde en ruine où elle rencontre le petit Brodeck. Elle présente toutes les caractéristiques d'une exilée, d'une étrangère en fuite perdue dans un monde qu'elle ne connaît pas. Lorsqu'elle rencontre Brodeck, perdu lui aussi dans ces ruines, dans ce "début d'une autre guerre » (Ibid.) elle le recueille. Ils ne se comprennent pas mais ils s'acceptent car ils ont besoin l'un de l'autre: "J'ai suivi la vieille femme comme si elle avait été un joueur de flûte. Elle m'a hissé sur la charrette, m’a calé entre des sacs, trois casseroles et un ballot de foin... Elle m'a demandé dans ma langue mon nom, m'a donné le sien » (Claudel, 2010: 28-29). Fédorine est étrangère pour Brodeck mais surtout étrange par sa façon de s'exprimer « des mots que je ne comprenais pas » (Claudel, 2010:28), de se déplacer « elle m'a hissé sur la charrette » (Claudel, $2010: 29)$ et par son univers : « il y avait un lapin aussi aux beaux yeux bruns et au poil fauve, dont le ventre était doux et très chaud » (Ibid.). Dans la détresse, dans sa perte de repères au milieu de ce conflit qui a détruit son village le petit Brodeck est fasciné par cette étrangeté proche du miracle, de la magie : " elle a fouillé dans sa besace pour en sortir une pomme d'un beau rouge luisant » (Claudel, 2010 : 28). Fédorine apparaît aux yeux de l'enfant comme une créature magique qui pourrait être sortie d'un conte. Brodeck est perdu au milieu de ces ruines et Fédorine le sauve : «Regarde bien petit Brodeck, tu viens de là et tu n'y retourneras plus car il ne restera plus rien de lui bientôt » (Claudel, 2010 : 29). Brodeck est sauvé par l'étrangère et son étrangeté, étrangeté de posséder de la nourriture feérique dans la pénurie, étrangeté 
d'avoir comme compagnon un lapin doux et chaud... Brodeck plonge dans l'étrangeté dès son enfance, étrangeté qu'il fera sienne en devenant le fils adoptif de Fédorine, en parcourant des milliers de kilomètres à ses côtés, intégrant peu à peu dans son propre univers l'enseignement de sa sauveuse dont le nom lui-même nous renvoie au mystère de sa personnalité, Fédorine qui devient une fée dorée pour le petit Brodeck :

Nous venions du bout du monde. Notre voyage avait duré des semaines, comme un rêve qui ne se terminait jamais. Nous avions traversé des frontières, des fleuves, des paysages, des cols, des villes, des ponts, des langues, des peuples, des forêts et des champs... Fédorine chaque jour me nourrissait de pain, de pommes et de lard, qu'elle tirait de grands sacs en toile bleue, et aussi de mots, des mots qu'elle glissait dans mon oreille et que je devais ressortir par ma bouche (Claudel, $2010: 70$ )

De cette étrangeté partagée naît une nouvelle communauté à bord de la charrette de Fédorine basée sur la compréhension mutuelle, sur la reconnaissance de l'autre à travers les souffrances endurcies dans le passé. Brodeck a besoin de Fédorine qui, elle-même, a trouvé en la personne de Brodeck le reflet de sa souffrance, le besoin d'affection, le tout donnant lieu à un début de construction, à la naissance d'une société :

Une mimésis sociale, une contagion émotionnelle sont souterrainement et continûment au travail, et contribuent à la construction identitaire des sujets sociaux. C'est le processus originaire, primaire, de l'identification qui se révèle être le socle ferme des constructions identitaires socialement compossibles, et donc de l'association humaine. Cette identification inconsciente de chacun, du Soi à un Autre, à sa ou à ses figures de prédilection, assure la cohésion et la cohérence de l'ensemble des Nous. Le phénomène de l'identification est au fondement du lien social, comme il est au fondement du lien œdipien, qui se révèle être la forme la plus primitive de l'attachement affectif (Farrugia, 2010 : par. 76)

8 Par cette communion tout au long de leur voyage disparaît l'étrangeté initiale, celle de Fédorine mais aussi celle de Brodeck, enfant perdu dans un monde en guerre.

\section{Brodeck ou la souffrance de par l'étrangeté}

9 L'étrangeté reparaît à l'issue de ce long voyage lorsque Fédorine et Brodeck arrivent dans un nouveau monde, un monde étranger, étrange car inconnu :

Des femmes sont venues nous apporter de quoi manger et de quoi boire. Je me souviens aussi du visage des hommes qui ont tiré la charrette et l'ont menée vers la cabane, ne voulant plus que Fédorine fasse le moindre effort. Puis il y eut le curé Peiper, qui était jeune et plein d'entrain... et puis le Maire, un vieil homme à grandes moustaches blanches et catogan, du nom de Sibelius Craspach (Claudel, $2010: 70)$

10 Accueilli par une collectivité où la peur ne règne pas encore: "C'était un temps où personne encore n'avait peur des étrangers même lorsqu'ils étaient les plus pauvres des pauvres » (Ibid.), Brodeck grandit dans cette société paisible où l'étrangeté s'estompe peu à peu, du moins en apparence. Mais Brodeck et Fédorine vivent seuls, à l'extérieur du village, dans la cabane cédée par la mairie dès leur arrivée. Ils continuent à développer cette communauté à deux, en marge du reste des habitants ils construisent leur propre bonheur :

On nous a installés dans la cabane en nous faisant comprendre que nous pouvions y rester une nuit ou des années... Les murs de bois avaient une douce et chaude couleur de miel. Il y faisait chaud. La nuit, on entendait parfois le murmure du vent dans les hautes branches des sapins tout proches et le craquement du bois caressé 
par le souffle du poêle. Je m'endormais en pensant aux écureuils, aux blaireaux et aux grives. C'était un paradis (Ibid.)

11 Puis les temps changent, la guerre arrive accompagnée de la peur de l'autre, de la persécution de l'étrangeté, de la condamnation de la différence.

Brodeck part à la ville faire des études et il assiste aux premières manifestations agressives envers l'étrange, envers l'étranger. Aucune date, aucun repère historique ne nous est donné mais nous pouvons librement resituer les évènements dans une période similaire à celle annonçant la seconde guerre mondiale. Les émeutes dont Brodeck est le témoin montrent la montée de la xénophobie au sein de milieux bien précis. Brodeck comprend alors que l'étrangeté peut être interprétée comme une menace par ceux qui ne la partagent pas :

Cette histoire de savoir et d'ignorance, de solitude et de nombre, c'est elle qui m'a fait quitter la ville, avant la fin de mes études. Il y eut soudain, pour agiter ce grand corps tentaculaire, des bruits, des rumeurs qui naissaient d'un rien, deux ou trois conversations, un article de quelques lignes, non signé, dans un quotidien, le boniment d'un bateleur sur un marché, une chanson venue de nulle part et dont le refrain féroce était repris en un clin d'œil par tous les chanteurs de rue (Claudel, $2010: 213)$

13 Il connaît alors le danger de ce qu'a désigné Pierre Bourdieu, sociologue français, comme le social individué :

La société [...] existe dans l'objectivité sous formes de structure sociales, de mécanismes sociaux [...] et elle existe aussi dans les cerveaux, c'est à dire dans les individus; autrement dit la société existe à l'état individuel, à l'état incorporé. Autrement dit, l'individu biologique socialisé, c'est du social individué. Cela dit, cela ne veut pas dire que le problème du sujet des actions ne se pose pas : est-ce que le sujet est conscient ou non? (Bourdieu, 1988 : par. 4)

Pour Brodeck c'est le début de la découverte de l'horreur déchaînée par la peur de l'autre, la peur de l'étrange, la haine de l'étranger.

Brodeck est finalement déporté, dénoncé par le reste des villageois terrorisés par une occupation qui les opprime, qui les anéantit. Ils dénoncent Brodeck pour sa différence du reste de la communauté, différence physique mais aussi pour le mystère qui entoure son arrivée, pour la langue qu'il parle avec Fédorine, pour son indépendance, en définitive, pour son étrangeté :

Chacun prit un petit morceau de papier pour y inscrire les noms des mauvais papillons. Je suppose que c'est le Maire qui ramassa les papiers et qui les lut. Tous les petits papiers comportaient deux noms, celui de Simon Frippman et le mien [...] Frippman et moi avions en commun de ne pas être nés au village, de ne pas ressembler à ceux d'ici, yeux trop sombres, cheveux trop noirs, peau trop bistre, de venir de loin, d'être d'un passé obscur et d'une histoire douloureuse, errante et séculaire... Simon Frippman et moi, nous étions donc des Fremdër - "pourritures » et «étrangers » - , les papillons que l'on tolère un temps, quand tout va bien, et qu'on offre en victimes expiatoires, quand tout va mal (Claudel, $2010: 277$ )

Brodeck constate que ni lui ni Fédorine n'ont jamais véritablement été acceptés au sein du village. L'emploi du verbe «tolérer » dénote un accueil forcé par une morale imposée dans un contexte social fermé ; les différences qui séparent Brodeck et sa mère adoptive du reste des habitants du village ne permettraient jamais une véritable intégration de par leurs origines étrangères inconnues. Leur passif et leur passé seraient toujours une immense muraille empêchant cette intégration de par le lien unissant émotions et 
contexte social dans les collectivités, tel que nous l'explique les théories de Maurice Halbwachs, sociologue français :

L'expression des émotions, mais à travers elle les émotions elles-mêmes sont pliées aux coutumes et aux traditions et s'inspirent d'un conformisme à la fois extérieur et interne. Amour, haine, joie, douleur, crainte, colère, ont d'abord été éprouvés et manifestés en commun, sous forme de réactions collectives. C'est dans les groupes dont nous faisons partie que nous avons appris à les exprimer, mais aussi à les ressentir [...] Par là, on peut dire que chaque société, chaque nation, chaque époque aussi met sa marque sur la sensibilité de ses membres. Sans doute il subsiste en ce domaine une large part de spontanéité personnelle. Mais elle ne se manifeste, elle ne se fait jour que dans des formes qui sont communes à tous les membres du groupe, et qui modifient et façonnent leur nature mentale aussi profondément que les cadres du langage et de la pensée collective (Halbwachs, 1947 : 10-11)

Lors de sa déportation Brodeck entre en contact avec la terrible violence de la race humaine, prête à tout pour faire disparaître l'étrange au sein de son univers. Brodeck vit alors l'humiliation, la souffrance causée par la haine profonde des hommes, leur peur de la différence, de l'étrangeté :

Je sais comment la peur peut transformer un homme. Je ne le savais pas, mais je l'ai appris. Au camp. J'ai vu des hommes hurler, se frapper la tête contre des murs en pierre, se lancer sur des fils de fer tranchants comme des rasoirs. J'en ai vu faire dans leur pantalon, se vider entièrement, vomir, sortir d'eux-mêmes tout ce qu'ils avaient de liquide, d'humeurs, de gaz. J'en ai vu certains prier et j'en ai vu d'autre renier le nom de Dieu, le couvrir de sanies et d'injures. J'ai même vu un homme en mourir. Mourir de peur, alors qu'un matin il venait d'être désigné au petit jeu des gardes comme le prochain à être pendu (Claudel, 2010 : 270)

Brodeck découvre que l'étrangeté peut devenir un danger pour celui qui en est la marque, lui-même en étant la victime.

Mais Brodeck revient de tout cet enfer et doit faire face à une nouvelle marque de la cruauté humaine dans le ventre de sa femme, violée pendant son absence. Brodeck cherche à comprendre, à survivre :

Pourquoi ai-je dû, comme des milliers d'autres hommes, porter une croix que je n'avais pas choisie, endurer un calvaire qui n'était pas fait pour mes épaules et qui ne me concernait pas? Qui a donc décidé de venir fouiller mon obscure existence, de déterrer ma maigre tranquillité, mon anonymat gris, pour me lancer comme une boule folle et minuscule dans un immense jeu de quilles? (Claudel, $2010: 238$ )

Les années passent et Brodeck rencontre cet étranger venu au village L'Anderer. Par cette rencontre Brodeck revivra l'horreur passée au travers de l'horreur présente dans le destin tragique de L'Anderer.

\section{L'Anderer ou la mise en abîme de l'étrangeté}

21 L'Anderer est immédiatement un étranger par la transcription même dans son nom de sa marque étrange, de son origine étrangère. L'Anderer, l'autre en allemand, est dès son arrivée condamné par son étrangeté qui sera plus tard accentuée par ses origines.

Dès les premières pages du livre de Philippe Claudel, le destin de Brodeck et celui de 1 'Anderer sont liés. Leurs trajectoires étranges, leurs arrivées au villages, leurs différences font que pour Brodeck, l'Anderer n'est pas tout à fait l'autre que veulent voir en lui le reste des villageois : «Mais pour moi, il a toujours été De Anderer - l'Autre -peut-être parce qu'en plus d'arriver de nulle part, il était différent, et cela, je connaissais bien : parfois 
même, je dois l'avouer, j'avais l'impression que lui, c'était un peu moi » (Claudel, 2010 : 12). Brodeck reconnaît en l'Anderer cette étrangeté qui fut la sienne, celle de Fédorine lorsqu'il la rencontra dans son enfance.

Lorsque Brodeck aperçoit l'Anderer pour la première fois il n'est plus l'enfant étonné par cette étrangeté mais l'adulte qui a souffert dans les camps pour en être lui-même porteur. Brodeck sait maintenant que toute cette différence qui séparait l'Anderer du reste des villageois est la cause du drame de sa mort.

Le livre de Philippe Claudel est construit de telle sorte que le passé et le présent sont sans cesse entremêlés et le va et vient entre le destin de Brodeck et celui de l'Anderer est constant afin de nous mettre en évidence à quel point l'étrangeté de leurs personnalités les réunit dans un malheur collectif commun.

Au tout début du livre (page 12), Brodeck nous fait part de sa peur face au reste des villageois : «je ne l'ai pas dit car je n'avais pas envie de finir comme l'Anderer » (Claudel, 2010 : 12). Cette peur est, nous le voyons déjà, liée au destin fatidique de l'Anderer. En citant les différents surnoms attribués à l'Anderer par le reste des habitants, Brodeck met en relief l'étrangeté de ce personnage au sein de cet univers fermé qu'est la communauté des villageois : «Vollaugä -Yeux pleins- [...]; De Murmelnër -le Murmurant- [...]; Mondlich Lunaire- [...] ; Gekamdörhin -celui qui est venu de là-bas. »(Claudel, 2010 :12).

Tout le livre est ponctué par l'étrangeté de l'Anderer, par la vision que les autres ont de lui. Ce récit nous est fait par le biais du rapport parallèle de Brodeck et c'est donc par le filtre de la peur grandissante de Brodeck que nous parvient ce crescendo vers la tragédie.

L'Anderer nous est présenté comme un être mystérieux pouvant accomplir ce que personne ne parvient à faire, comme caresser le chien féroce de Göbbler, un voisin menaçant de Brodeck :

Alors l'Anderer s'arrêtait, enlevait ses gants, de jolis gants de cuir très fin et très souple, et lui caressait le crâne. Et c'était étrange de les voir ainsi tous les deux, le chien placide et heureux, qui acceptait la caresse sagement alors que d'ordinaire aucun de nous ne pouvait l'approcher vraiment et encore moins le toucher (Claudel, 2010: 35)

L'adjectif étrange dans les propos de Brodeck semble déjà être un indice de la différence de l'Anderer.

Par la suite, lorsque Brodeck revient sur l'arrivée de l'Anderer la peur transparaît à nouveau dans ses propos: «Il faut que je raconte l'arrivée de l'Anderer, chez nous, mais j'ai peur : peur d'agiter des fantômes, et peur des autres » (Claudel, 2010 : 59). C'est la peur grandissante en présence de l'étrangeté qui réapparaît moins d'un an après la fin de la guerre. Tout est trop proche, tout est trop étrange, cet homme dont on ne connaît pas le nom, ses animaux, son comportement. Lorsqu'il en parle Brodeck retranscrit les impressions d'un des habitants du village qui laisse déferler dans ses propos tous les préjugés qui finalement seront la cause de la mort de l'Anderer: "C'était comme une apparition d'une autre époque. J'en suis resté bouche bée. Un vrai personnage de foire, attifé comme on ne fait plus, et trottinant avec ses montures de cirque comme s'il allait à la revue ou sortait d'un théâtre de marionnettes » (Claudel, $2010: 62$ ).

Lorsque Brodeck entre en contact avec l'Anderer il va comprendre que leurs points communs vont au-delà de leur destin. Brodeck partage avec l'Anderer un intérêt pour la botanique, pour la vie naturelle, pour la beauté de la nature : «On m'a dit que vous vous intéressiez aux fleurs, aux herbes. Nous nous ressemblons. Je suis un amateur de 
paysages, de figures et de portraits » (Claudel, $2010: 111)$. L'Anderer représente aux yeux de Brodeck ce que Francis Farrugia dénomme la déprise: "Cette déprise qui est d'une certaine manière un éveil, génère une rupture existentielle, une conversion du regard et une inversion du rapport d'adhésion au sentiment commun, à une convention collective désormais mise à distance comme senti artificiel collectivement construit " (Farrugia, 2010 : 38).

Brodeck partage aux côtés de l'Anderer un univers étrange mais qui ne lui est pourtant pas étranger. À ses côtés il prend conscience de ce que fût son passé, son expérience de la différence :

J'ai dit que l'Anderer avait un sourire qui ne semblait pas appartenir à notre monde. Mais c'est tout simplement parce que lui-même n'était pas de notre monde. Il n'était pas de notre histoire. Il n'était pas dans l'Histoire. Il arrivait de nulle part et aujourd'hui il n'y a plus de trace de lui, c'est comme s'il n'avait jamais existé. A qui donc, mieux qu’à lui, pouvais-je raconter? Il n'était d'aucun côté (Claudel, 2010 : 287)

Il acquiert une certaine lucidité quant à la cruauté des hommes, il s'éloigne de plus en plus de la vision commune qui fut celle qui causa son exil dans les camps. Cette lucidité naissante aux côtés de l'Anderer ressurgira dans la rédaction de son rapport parallèle dans lequel il tente de recomposer les vides de son existence par l'analyse de la tragédie dont l' Anderer fut la victime. S'éloigner de la vision collective, juger par soi-même, Francis Farrugia nous l'explique ainsi :

Cette lucidité prend racine dans un système émotionnel et sentimental non construit de manière orthodoxe, non imprégné de la doxa commune des sentis et ressentis conventionnels [...] Cette connaissance directe de la vérité du monde senti et vécu (le Lebenswelt des phénoménologues) dans sa nudité première, dans son étrangeté, est clairement l'essence même du regard philosophique porté sur les choses et les hommes (Farrugia, $2010: 38$ )

Cet éloignement de la vision commune causera la perte de l'Anderer. Son point de vue si présent dans le tracé des portraits qu'il peint des villageois déchaînera la colère de ces derniers qui finiront par l'assassiner, éveillant en eux la peur que cet étranger ne découvre leur passé commun, leurs trahisons, leurs crimes : « Pour tous les visages, il en était de même. Les portraits qu'en avait faits l'Anderer agissaient comme des révélateurs merveilleux qui amenaient à la lumière les vérités profondes des êtres. On aurait cru une galerie d'écorchés » (Claudel, $2010: 325)$. Se poursuit alors la spirale de la peur de l'autre et l'Anderer est assassiné par les villageois méfiants et cruels. Brodeck devient à nouveau témoin de la violence que déchaînent chez les hommes la peur de l'étrangeté, le rejet de la différence, comme il le fut dans sa propre chair, dans son passé proche, pendant son internement dans les camps.

\section{Conclusion}

Dans le cadre de l'écriture de son double rapport, Brodeck revient sur son terrible passé et la cruauté des hommes effrayés par la présence de l'autre devient alors son fil conducteur.

Partant du massacre de l'Anderer par les villageois qui l'entourent, il se souvient de ce que fut la découverte et l'expérience de l'étrangeté depuis sa plus tendre enfance. Il revient sur son sauvetage par Fédorine et sur son initiation au monde étrange par la bonté et le 
partage que lui offrit sa mère adoptive. Mais la progression dans ce monde de la différence le replonge dans son terrible vécu au sein de l'horreur des camps de concentration. Dénoncé à l'occupant par les notables du village pour ses «yeux trop sombres, cheveux trop noirs, peau trop bistre » (Claudel, 2010 : 277) il connut alors le prix de la différence, le coût de l'étrangeté.

La re-connaissance de l'étrangeté en la personne de l'Anderer le guide vers une lucidité qui peu à peu l'aide à se libérer de son passé et de la peur de sa propre étrangeté. La déprise dont il fait l'expérience représente une rupture existentielle qui l'aide à fuir un monde où le regard collectif mène au crime, pour se diriger vers un monde où le passé n'existe plus. Ainsi Brodeck repart comme il était venu, emmenant avec lui ses êtres chers et laissant derrière lui ce passé enfin oublié et maintenant si lointain : "C'était comme si le paysage et tout ce qu'il avait contenu s'étaient effacés derrière mes pas. Comme si à mesure que j'avançais, on avait démonté le décor, plié les toiles peintes, éteint les lumières » (Claudel, 2010 : 374).

\section{BIBLIOGRAPHIE}

BOURDIEU, Pierre (1988). « Pierre Bourdieu - L'habitus est un système de virtualité qui ne se révèle qu'en situation : entretien avec Roger Chartier, diffusé sur France Culture dans le cadre de l'émission Les chemins de la connaissance, (partie 4) » [retranscrit sur le site Sociotoile] [on-line] [disponible le 20 octobre 2011] <http://www.sociotoile.net/article51.html\#nb1>

CLAUDEL, Philippe (2010). Le rapport de Brodeck. Paris : Le livre de poche.

FARRUGIA, Francis (2010). « Le syndrome narratif : une inquiétante étrangeté » [on-line] in Sociologies (Dossiers, Émotions et sentiments, réalité et fiction) [disponible le 20 octobre 2011] < http://sociologies.revues.org/3152>

FUGIER, Pascal (2006). « Un inquiétant sentiment d'étrangeté au principe d'une sociologie hétérodoxe » [on-line] in ¿Interrogations? - Revue pluridisciplinaire en sciences de l'homme et de la société, no 2 : La construction de l'individualité. [disponible le 20 octobre 2011] <URL : http:// www.revue-interrogations.org>

HALBWACHS, Maurice (1947). « L'expression des émotions et la société » [on-line], in Échanges sociologiques [disponible le 20 octobre 2011] <http://classiques.uqac.ca/classiques/ Halbwachs_maurice/classes_morphologie/partie_2/texte_2_4/expression_emotions.html>

\section{RÉSUMÉS}

Dans Le Rapport de Brodeck Philippe Claudel aborde le thème du regard de la communauté face à l'étranger. Dans un village frontalier, un habitant du village, Brodeck, est chargé contre son gré par le reste de la communauté villageoise de rédiger un rapport sur un évènement dramatique. À travers l'écriture forcée de ce rapport sur le lynchage d'un étranger par le reste des villageois, Brodeck se replonge dans son passé proche, dans sa souffrance de l'univers des camps, dans sa 
propre expérience de la xénophobie. C'est alors que dans l'écriture de Brodeck, le texte anonyme rejoint la confession intime et nous guide vers la douleur de l'indicible, vers son apaisement par l'écriture de la survie. Dans notre article nous tenterons de montrer comment Philippe Claudel construit dans ce récit intemporel, dans cette reconstitution anonyme des faits qu'est Le Rapport de Brodeck un véritable plaidoyer contre la xénophobie.

In Brodeck's Report, Philippe Claudel addresses the theme of the regard of a community towards the alien. In a small border town, a local resident, Brodeck, is charged against his will by the rest of the community to prepare a report on a dramatic event. By means of this forced writing about the lynching of a foreigner by the rest of the villagers, Brodeck plunges back into his recent past, in the suffering of the prisoners' camps in his own experience of xenophobia. It is then that in Brodeck's writing, anonymous text and intimate confession are joined and take us towards the unspeakable pain, to appease it through the writing of survival. In our article we will attempt to show how Philippe Claudel built in this timeless tale, in this anonymous reconstruction of the facts, what the Brodeck's Report is: a plea against xenophobia.

\section{INDEX}

Keywords : Claudel (Philippe), strangeness, foreign, xenophobia, abandonment

Mots-clés : Claudel (Philippe), étrangeté, étranger, xénophobie, déprise

\section{AUTEUR}

DOMINIQUE BONNET

Universidad de Huelva

domi[at]uhu.es 